\title{
Cryopreservation of human whole blood for pyrogenicity testing
}

\author{
Stefanie Schindler, Silvia Asmus, Sonja von Aulock, Albrecht Wendel, \\ Thomas Hartung*, Stefan Fennrich \\ Biochemical Pharmacology, University of Konstanz, D-78457 Konstanz, Germany
}

\begin{abstract}
Human whole blood assays are increasingly employed to test immune function or detect pyrogenic contamination, since they offer advantages, such as ease of performance, few preparation artifacts and a physiological cell environment. However, the approach is often limited by the availability of freshly drawn blood, putative safety concerns in the case of infected donors and interindividual donor differences. To overcome these limitations, a method was developed and optimized to produce batches of cryopreserved blood that can be used directly after thawing without any washing steps. Mononuclear cells remained intact as shown by FACS analysis. Cytokine release could be induced by a variety of immunological stimuli. The cell preparation released higher amounts of interleukin-1 $\beta$ (IL-1 $\beta$ ) and IL-6 compared to fresh blood, but no TNF. These differences could be attributed to the presence of the cryoprotectant dimethylsulfoxide (DMSO) alone by addition of DMSO to fresh blood. Large batches of cryopreserved blood could be produced by mixing blood donations of up to 10 donors, independent of differing blood groups. The detection limit for the World Health Organization (WHO) lipopolysaccharides (endotoxin, LPS) reference preparation (EC-6) with regard to the induction of IL-1 $\beta$ release was at least 0.5 endotoxin equivalent units $(\mathrm{EU}) / \mathrm{ml}$. Endotoxin spikes at the limit concentrations prescribed in the European Pharmacopoeia could be detected in a series of drugs, showing that the In vitro Pyrogen Test (IPT) can also be run with cryopreserved blood. Further possible applications include high-throughput screening for immunomodulators or toxins as well as preservation of patient samples for later analysis of cell functions.
\end{abstract}

Keywords: Cryopreservation; Blood; Endotoxin; Interleukin-1 $\beta$; In vitro Pyrogen Test (IPT)

Abbreviations: DMSO, dimethylsulfoxide; ELC, endotoxin limit concentration; EU, endotoxin equivalent units; IL-1 $\beta$, interleukin-1 $\beta$; LPS, lipopolysaccharide, endotoxin; LTA, lipoteichoic acid; MVD, maximal valid dilution; RT, room temperature; WHO, World Health Organization.

* Corresponding author. Tel.: +49 753188 4116; fax: +49 7531 884117.

E-mail address: thomas.hartung@uni-konstanz.de (T. Hartung).

\section{Introduction}

Cryopreservation of cells represents a standard procedure in cell culture. Human primary leukocytes are cryopreserved on a routine basis, for example, to store human bone marrow cells (Goldman et al., 1978). Further cryopreservation protocols have been established for various isolated blood cell populations, 
including lymphocytes and mononuclear cells, and the retention of various cell functions after thawing has been investigated (Cavins et al., 1965; Knorpp et al., 1967; Handin and Valeri, 1972; De Boer et al., 1981; Frim and Mazur, 1983).

Although it is popular to isolate the respective immune cells from blood, it is evident that such isolated cells do not reflect the in vivo situation. For example, the cells are often stimulated during the isolation procedure as indicated by basal mediator release or adherence of the cells. Moreover, interactions between different cell types cannot take place and plasma components that often play an important role in immune recognition are no longer present.

Methods employing whole blood have been developed to detect pyrogenic (fever-inducing) contamination, e.g., of batches of injectable drugs (Hartung and Wendel, 1995). This application has been successfully validated in a collaborative European study and awaits incorporation into the pharmacopoeias (Hartung, 2002). Furthermore, we have suggested the study of cytokine, histamine or eicosanoid release with this method to facilitate the characterization of putative drugs or immunotoxins (Hermann et al., 2003; von Aulock et al., 2003). These methods can also be used ex vivo on treated volunteers or patients to monitor the course and effects of treatment (Hartung et al., 1995, 1999; von Aulock et al., 2000).

Many of these procedures could be simplified or optimized by the availability of cryopreserved whole blood. The blood could be supplied in the form of a standardized test reagent which could be stored until needed and be certified free of infectious agents. A method to preserve and store cells from treated patients might allow performance of the often laborious cell assays on a series of samples collected in parallel or at a distant laboratory, thus reducing variability and logistical problems.

We sought to develop a protocol which would allow the use of the thawed whole blood samples directly without any washing steps to remove the cryoprotectant, as such a step would eliminate the essential advantages of the human whole blood assay, i.e., the ease of performance which allows a high degree of standardization as shown for various applications (Fennrich et al., 1999). Furthermore, besides stress and handling artifacts, the cells would lose their autologous plasma that permits a number of physiological responses, e.g., the sensitive response to lipopolysaccharides (endotoxin, LPS) via lipopolysaccharide binding protein (LBP; Schumann, 1992; Fenton and Golenbock, 1998).

In this report, we describe the development of a protocol to freeze whole human blood and demonstrate the retention of sensitivity and functionality regarding stimulation of cytokine release in response to inflammatory agents.

\section{Materials and methods}

\subsection{Freezing procedure}

Blood was drawn from healthy volunteers into tubes containing $15 \mathrm{IU} / \mathrm{ml} \mathrm{Li-Heparin} \mathrm{(Sarstedt,}$ Nürnbrecht, Germany) and differential blood cell counts were performed on each sample to rule out active infections (Pentra 60, ABX Diagnostics, Montpellier, France). In order to rule out pyrogenic contaminations of any component used in the incubations, negative saline controls were included in each experiment. The heparinized blood was precooled in ice water for $15 \mathrm{~min}$. Clinical grade dimethylsulfoxide (DMSO, Waco Chemicals, Dessau-Thornau, Germany) was added to the blood in $50-\mathrm{ml}$ centrifugation tubes (Greiner bio-one, Frickenhausen, Germany) in small amounts to a final concentration of $10 \%$ ( $\mathrm{vol} / \mathrm{vol}$ ratio) under constant gentle agitation to avoid cell damage. Pooling was performed in 50-ml centrifugation tubes after the addition of DMSO to the blood of the individual donors. Blood was pipetted as 1,3 or 4 $\mathrm{ml}$ aliquots into precooled cryotubes $(1.8,3.6$ or 4.5 $\mathrm{ml}$, Nunc, Wiesbaden, Germany) and put into the rack of a programmable freezer with a TP type nitrogen container (Nicool Plus PC, Air Liquide, Marne-laVallée Cedex 3, France), precooled to $4{ }^{\circ} \mathrm{C}$. A temperature probe was inserted into an extra aliquot containing the same volume of blood to follow the freezing process. The freezing program was started 5 min after closing the freezer. The blood was cooled down to $-5{ }^{\circ} \mathrm{C}$ at a rate of $1{ }^{\circ} \mathrm{C} / \mathrm{min}$. In order to compensate for the latent heat of fusion generated by the blood when changing from the liquid to the solid state. The temperature Tx in the freezing chamber was set to $-30{ }^{\circ} \mathrm{C}$. The crystallization temperature was 
$-12{ }^{\circ} \mathrm{C}$. When this temperature was reached, the blood was cooled down to $-40{ }^{\circ} \mathrm{C}$ at a rate of $2{ }^{\circ} \mathrm{C}$ / $\mathrm{min}$. The blood was given $120 \mathrm{~s}$ to stabilize before being cooled down to $-120^{\circ} \mathrm{C}$ at a rate of $10^{\circ} \mathrm{C} / \mathrm{min}$. After freezing, the tubes were removed from the freezer and transferred immediately into the vapor phase of liquid nitrogen (nitrogen tank, Air Liquide, Kryotechnik, Düsseldorf, Germany).

\subsection{Thawing procedure}

The closed tubes were left in an incubator at $37{ }^{\circ} \mathrm{C}$ until completely thawed. The aliquots of single donors were either pooled or the blood was pipetted individually from each aliquot. Pooling of the blood of different donors could be performed after thawing as an alternative to the procedure described above. The whole blood incubation was started not more than $30 \mathrm{~min}$ after complete thawing.

\subsection{Whole blood incubation}

Human whole blood incubations were performed according to the protocol of the In vitro Pyrogen Test (IPT; Hartung and Wendel, 1995; Hartung, 2002) using either fresh or cryopreserved blood. Briefly, 100 $\mu l$ of fresh or cryopreserved human blood was added to $1 \mathrm{ml}$ physiological, clinical grade, pyrogen-free saline in polypropylene reaction tubes (Eppendorf, Hamburg, Germany). After the addition of stimuli, the tubes were closed, shaken gently and incubated overnight $(16-24 \mathrm{~h})$ at $37{ }^{\circ} \mathrm{C}$. The cells were resuspended and assayed immediately or frozen until measurement. Within each experiment performed, all samples were incubated and measured in parallel. When all samples of an experiment were measured on the same ELISA plate, absorbance $\left(A_{450}\right)$ was given as the unit of measurement according to the IPT protocol. When the samples could not be measured on the same ELISA plate, a recombinant standard curve was run on each ELISA plate to allow interplate comparison.

Endotoxin stimuli were LPS from Escherichia coli O113 [World Health Organization (WHO) standard material], kindly provided by Dr. Stephen Poole, NIBSC, Hertfordshire, GB, or LPS from E. coli O111 (IPT Kit, Charles River Endosafe, Charleston, SC, USA) calibrated to the WHO standard material. One important criterion for the In Vitro Pyrogen Test (IPT) was the ability to detect reproducibly the presence of 0.5 endotoxin equivalent units (EU) per $\mathrm{ml}$, equivalent to $50 \mathrm{pg} / \mathrm{ml}$ of the WHO reference endotoxin standard or to $100 \mathrm{pg} / \mathrm{ml}$ of the LPS from E. coli O111, respectively, in a sample solution, this being the fever threshold of the most sensitive rabbit strain if applied at a dose of $10 \mathrm{ml} / \mathrm{kg}$. Therefore, this LPS concentration was included in every assay.

Nonendotoxin stimuli were lipoteichoic acid (LTA) from Bacillus subtilis (IPT Kit, Charles River Endosafe; Morath et. al., 2002), glucan standard (Charles River Endosafe), glucan from barley (Sigma, Munich, Germany), lectin from Phaseolus vulgaris (PHA-L and PHA-E, Sigma), curdlan (Sigma) and zymosan A (Fluka, Buchs, Switzerland).

Substances tested at maximal valid dilution (MVD) were furosemide (Lasix ${ }^{\circledR}$ ), ampicillin $\left(\right.$ Binotal $\left.^{\circledR}\right)$, articain/epinephrine (Ultracain ${ }^{\circledR}$; Aventis, Germany), theophylline (Bronchoparat ${ }^{\circledR}$; Fujisawa, Munich, Germany), dimethindenmaleate (Fenistil ${ }^{\circledR}$; Novartis, Munich, Germany), ranitidine (Sostril ${ }^{\circledR ;}$; Glaxo Smith Kline, Munich, Germany) and metoprolotartrate (Beloc ${ }^{\circledR}$; Astra Zeneca, Wedel, Germany).

Cytokine ELISAs were based on commercially available antibody pairs against interleukin-1 $\beta$ (IL$1 \beta$ ) or TNF $\alpha$ (Endogen, Biozol, Eching, Germany), and IL-6 (R\&D, Wiesbaden, Germany). Binding of biotinylated antibody was quantified using streptavidin-peroxidase (Biosource, Camarillo, CA, USA) and the substrate TMB (3,3',5,5'-tetramethylbenzidine, Sigma). Recombinant cytokines serving as standards were gifts from Dr. S. Poole, NIBSC.

\subsection{FACS analysis}

Twenty-five microliters of fresh or cryopreserved blood was stained with $5 \mu$ each of anti-CD45-APC and anti-CD14-FITC antibodies (BD Biosciences, Heidelberg, Germany) for $30 \mathrm{~min}$ at room temperature (RT) in the dark. One milliliter of CellWash and propidium iodide in a final concentration of $500 \mathrm{ng} / \mathrm{ml}$ were added directly, immediately before measurement in a FACSCalibur (all BD Biosciences). A live gate was set on CD45-positive cells and 3000 leukocytes were counted. Whole blood counts were determined by Türks staining and counting in a Neubauer chamber. 


\subsection{Statistics}

Statistics were performed with GraphPad InStat 3.0 (GraphPad Software, San Diego, USA). Significance was tested by one-way ANOVA and Dunnett's posttest/Dunn's multiple comparison and with $t$-test, followed by Mann-Whitney posttest.

\section{Results}

\subsection{Freezing procedure}

Different concentrations of the cryoprotectant DMSO were tested to determine a concentration that would protect the cells and leave them functional after thawing but which would be sufficiently low to have no toxic effects in the incubation (Fig. 1). While 1\% DMSO was insufficient to protect the cells during freezing and $20 \%$ DMSO had toxic effects in the subsequent incubation period, cell preparations frozen with either $5 \%$ or $10 \%$ DMSO responded to stimulation with LPS in a concentration-dependent manner after thawing.

We compared whether the reactivity of the cryopreserved blood measured as the IL-1 $\beta$ response to endotoxin stimulation was affected by the blood

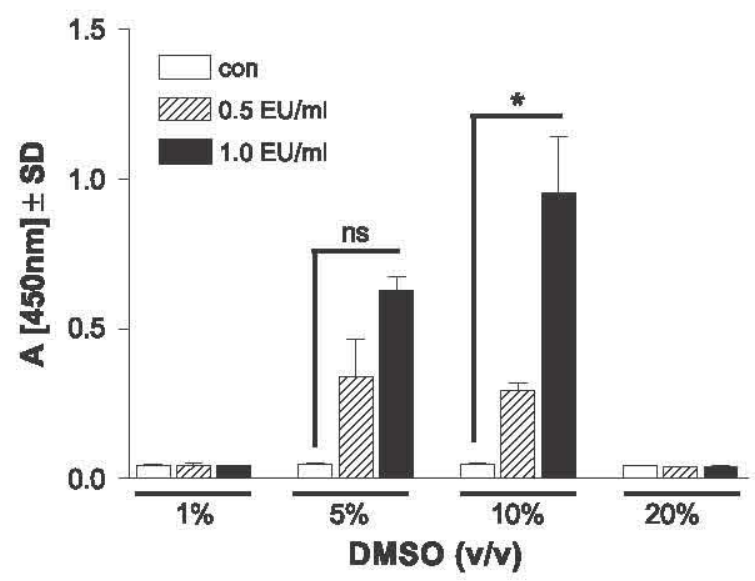

Fig. 1. Optimization of the final DMSO concentration in cryopreserved blood. Blood was frozen with different concentrations of DMSO as shown and stimulated with LPS from E. coli 0113 overnight after thawing (representative experiment of two). $\amalg L-1 \beta$ was measured by ELISA and is given as absorbance value \pm S.D. Four replicates of blood from one donor are shown, ${ }^{*} p<0.05$ (oneway ANOVA, posttest: Dunn's multiple comparison). temperature (room temperature or $4{ }^{\circ} \mathrm{C}$ ) at which the DMSO was added and whether DMSO should be added as a bolus or in several aliquots. The addition of DMSO at room temperature seemed to cause an increase in reactivity rather than a decline and addition of DMSO in several aliquots was preferable to the bolus (response to $0.5 \mathrm{EU} / \mathrm{ml}$ LPS O113 in OD: DMSO added at $4{ }^{\circ} \mathrm{C}$ as a bolus, $0.404 \pm 0.056$; DMSO added at $4{ }^{\circ} \mathrm{C}$ in three aliquots, $0.547 \pm 0.034$; DMSO added at room temperature as a bolus, $0.596 \pm 0.049$; DMSO added at room temperature in three aliquots, $0.728 \pm 0.051, p<0.01$, six to eight replicates of blood from one donor).

Next, we determined how long the blood could be kept after addition of DMSO before freezing and whether room temperature or $4{ }^{\circ} \mathrm{C}$ is preferable. For this purpose, DMSO was added to the blood and an aliquot was frozen immediately while other aliquots were stored at room temperature or at $4{ }^{\circ} \mathrm{C}$ for up to $200 \mathrm{~min}$ and then frozen and tested in parallel (Fig. 2). These data suggest that storage at room temperature for up to $2 \mathrm{~h}$ is tolerable and that storage at $4{ }^{\circ} \mathrm{C}$ is beneficial when the blood is stored for longer.

\subsection{Comparison of different volumes}

Different volumes of blood (1, 3 and $4 \mathrm{ml}$ aliquots) were frozen and stimulated with endotoxin after thawing. The reactivity of the blood did not depend on the volume of the frozen aliquots. The mean absorbance of the 1,3 , and $4 \mathrm{ml}$ aliquots when stimulated with $1.0 \mathrm{EU} / \mathrm{ml}$ of $\mathrm{O} 113$ was $2.824 \pm 0.066$, $2.463 \pm 0.058$ and $2.600 \pm 0.087$, respectively, in eight replicates of blood from one donor.

\subsection{Thawing procedure}

A thawing protocol was developed in order to optimize the handling of the blood aiming at maximum reactivity and viability. Aliquots of blood from the same donors were thawed under different conditions, i.e., on ice, at room temperature $\left(20^{\circ} \mathrm{C}\right)$ and in an incubator $\left(37^{\circ} \mathrm{C}\right)$ until completely thawed before stimulation with endotoxin (Fig. 3). Quick thawing at $37^{\circ} \mathrm{C}$ resulted in the best response.

An important issue was the potential cytotoxicity of the remaining cryoprotectant DMSO after thawing and before dilution with saline. Therefore, we tested 


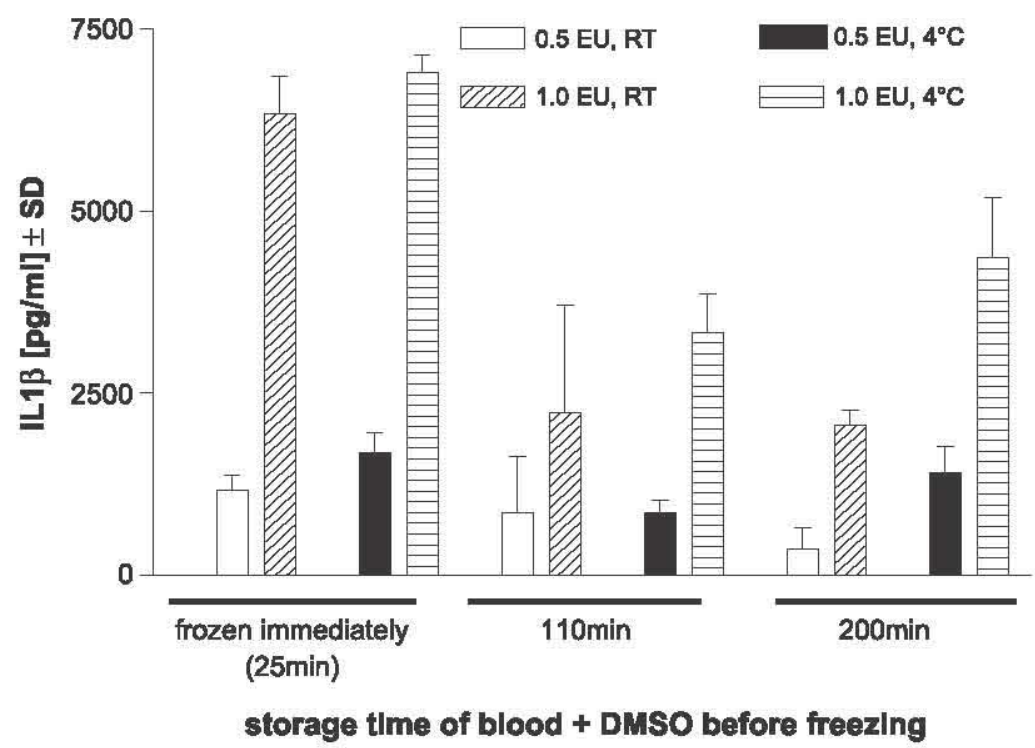

Fig. 2. Comparison of different storage temperatures and durations before freezing. Blood with $10 \%$ DMSO was frozen immediately or stored as indicated before freezing, then stimulated with LPS from $E$. coli O111 overnight after thawing (representative experiment of two). $L-1 \beta$ was measured by ELISA and is given as $\mathrm{pg} / \mathrm{ml} \pm$ S.D. Control values were $<6 \mathrm{pg} / \mathrm{ml} \mathrm{IL}-1 \beta$ for each condition. Four replicates of blood from one donor are shown $(0.5 \mathrm{EU} / \mathrm{ml}: n=5)$.

how long the thawed blood samples could be kept at $37{ }^{\circ} \mathrm{C}$ before dilution and stimulation (Fig. 4). The reactivity of the blood towards the endotoxin stim-

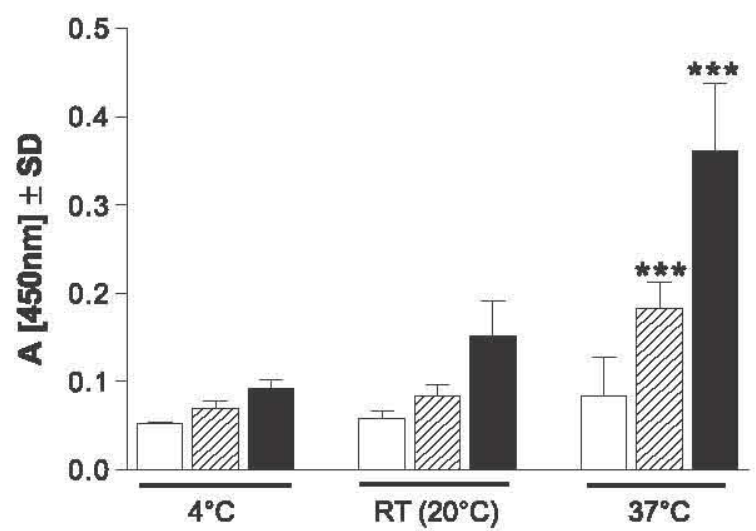

Fig. 3. Determination of a suitable thawing temperature. Cryopreserved blood with $10 \%$ DMSO was thawed at different temperatures $\left(4{ }^{\circ} \mathrm{C}\right.$, room temperature, $37^{\circ} \mathrm{C}$ ), then stimulated with $0.5 \mathrm{EU} / \mathrm{ml}$ (hatched bars) and $1.0 \mathrm{EU} / \mathrm{ml}$ (black bars) LPS from $E$. coli $\mathrm{O} 113$ or saline (white bars) overnight (representative experiment of four). IL$1 \beta$ was measured by ELISA and is given as absorbance value \pm S.D. Eight replicates of blood from one donor are shown. ${ }^{* * *} p<0.001$ against the respective stimulation after thawing at 4 or $20^{\circ} \mathrm{C}$ (oneway ANOVA with Dunnett's posttest). ulation decreased after $45 \mathrm{~min}$ of thawing time. Therefore, the blood was used within $30 \mathrm{~min}$ after thawing at $37{ }^{\circ} \mathrm{C}$ in all subsequent experiments.

\subsection{FACS analysis}

Differential blood cell counts were performed on parallel samples of fresh and cryopreserved blood from five donors. Although the whole blood cell counts of the cryopreserved blood did not differ from those of the fresh blood samples, the differential blood cell count revealed that the neutrophilic granulocytes had lost their surface markers and could no longer be identified as live, CD45 positive cells. The ratio of monocytes to lymphocytes in the differential blood cell count was the same in the fresh and the cryopreserved blood (1:6.7 \pm 0.9 versus $1: 8.1 \pm 1.6$, n.s.) with a viability of these two populations of $99 \%$ vs. $90 \%$ as shown by propidium iodide exclusion.

\subsection{Establishment of a pooling protocol}

Blood samples from five different donors were compared with each other and with pools of blood 


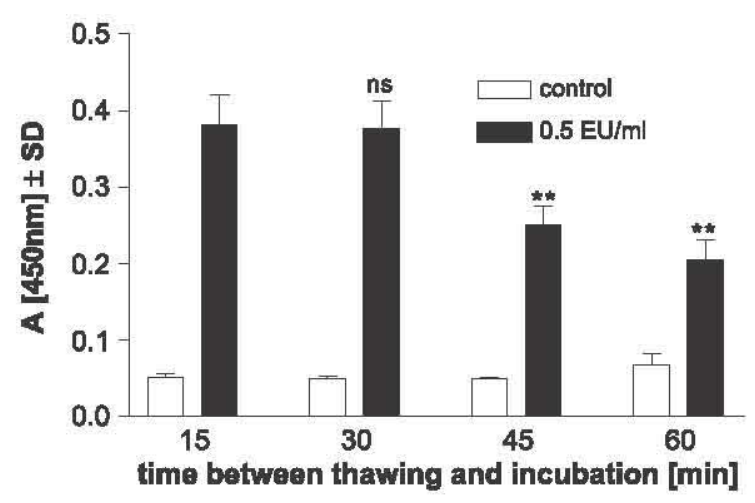

Fig. 4. Effect of time between thawing of blood and incubation. Blood was thawed and stored at $37^{\circ} \mathrm{C}$ for the times indicated, then stimulated with $0.5 \mathrm{EU} / \mathrm{ml}$ LPS from $E$. coli 0113 overnight (representative experiment of four). IL-1 $\beta$ was measured by ELISA and are given as absorbance value \pm S.D. Blood from one donor in five replicates is shown. ${ }^{* *} p<0.01$ vs. the values at $15 \mathrm{~min}$ (oneway ANOVA with Dunnett's post test).

from the same donors combined either directly after addition of the DMSO or after the thawing of frozen blood. Establishing a pooling protocol with blood from different donors with different blood groups proved easier than anticipated (Fig. 5). There was no difference in the reactivity of the blood pools, whether they were made before or after freezing.
In addition, the reaction of the pooled blood was equal to the mean of the reaction of the individual donors.

\subsection{Interlot variability}

The IL- $1 \beta$ response to endotoxin was compared between five different pools of cryopreserved blood, each containing the blood of five donors (Fig. 6). The interlot variability was very low, indicating that the use of five donors in the pooling protocol is sufficient to produce highly similar batches of blood.

\subsection{Stability}

Numerous aliquots of a pool of blood from five donors were frozen and their reactivity tested on different days over a period of 4 months. The IL-1 $\beta$ response to $0.5 \mathrm{EU} / \mathrm{ml}$ endotoxin was significantly different from the saline controls at each of the time points tested, indicating that the cryopreserved blood remained stable over this time period and did not lose sensitivity (Table 1).

To determine interaliquot variability of aliquots from the same blood donor, eight replicates each from 3 aliquots of $1 \mathrm{ml}$ thawed cryopreserved blood were stimulated with $0.5 \mathrm{EU} / \mathrm{ml}$ endotoxin and eight

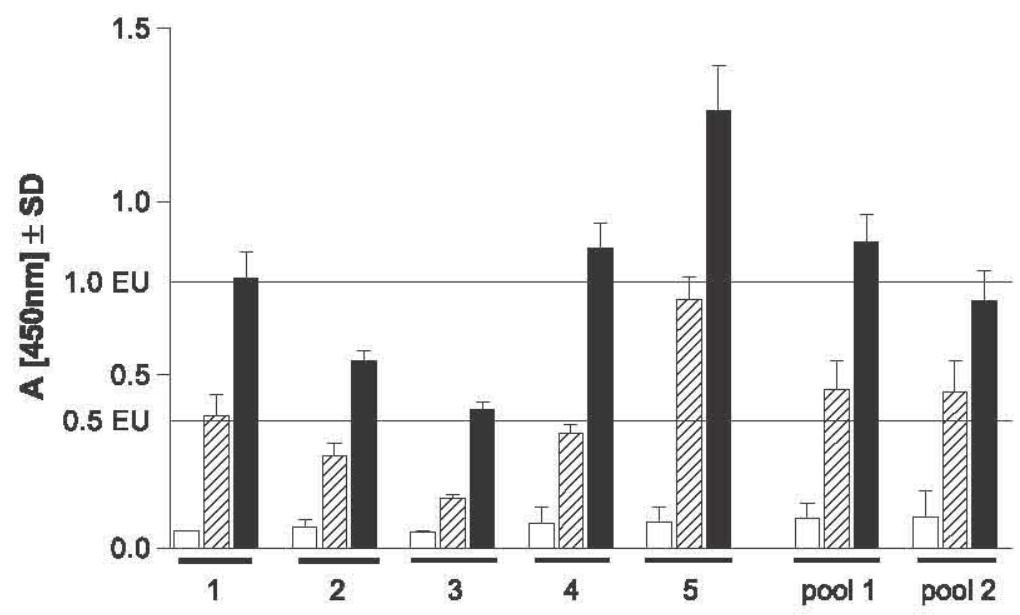

Fig. 5. Comparison of the reactivity of frozen blood from five donors and their pooled blood. Blood from five separate donors as well as a pool of their blood was frozen and stimulated as shown (representative experiment of four). The horizontal lines indicate the calculated mean of the blood from the five donors to 0.5 or $1.0 \mathrm{EU} / \mathrm{ml}$ LPS from $E$. coli $\mathrm{O} 113$ overnight. IL- $1 \beta$ measured by ELISA is given as absorbance value \pm S.D. Three replicates of all samples were measured $(0.5 \mathrm{EU} / \mathrm{ml}$ : four replicates). Pool 1 , the blood of the single donors was pooled after addition of DMSO; pool 2, the blood was pooled after thawing. 


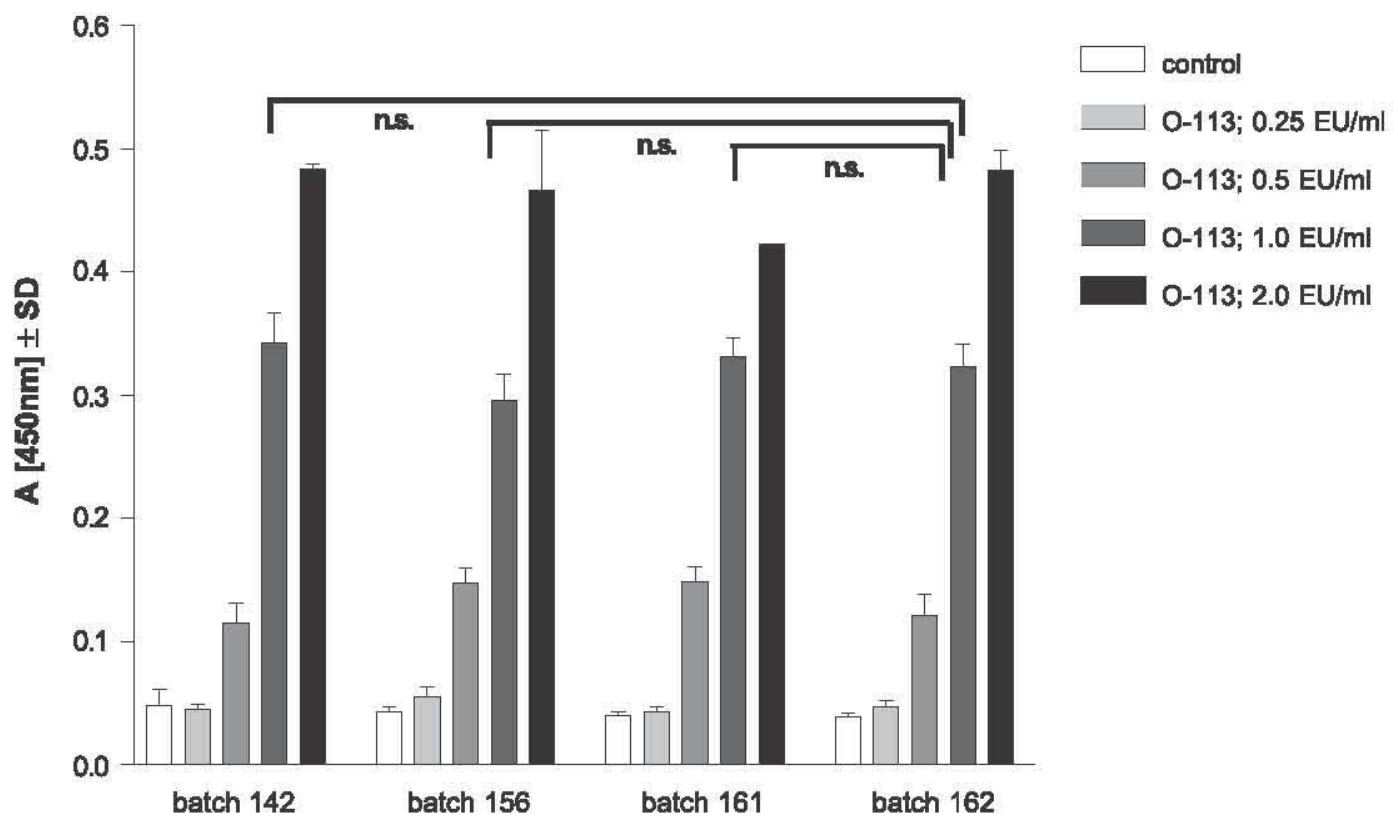

Fig. 6. Interlot variability of five different pools Cryopreserved pools each consisting of five different donors and frozen over a period of 23 weeks were thawed and stimulated on the same day $(0.25$ and $2 \mathrm{EU} / \mathrm{ml}$ : two replicates each; control, 0.5 and $1 \mathrm{EU} / \mathrm{ml}$ : four replicates each) with

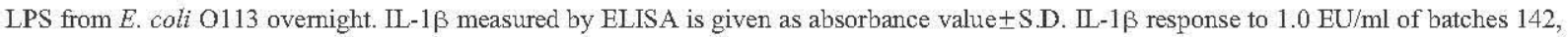
156 and 161 was not significantly different from that of batch 162 (one-way ANOVA, Dunnett's posttest).

replicates each from three aliquots were left unstimulated (Table 2). The stimulated samples had mean absorbance values of $0.27-0.49$ and the coefficient of variation (cv) was $12.3-26.1 \%$, while the unstimulated samples had mean values ranging from 0.047 to 0.054 and a cr of $5.4-42.2 \%$.

The interaliquot variability of the same experiment was $0.051 \pm 0.004$ (cv $7.3 \%$ ) for unstimulated versus $0.37 \pm 0.11$ (cv $31.3 \%$ ) for blood stimulated with 0.5 EU/ml LPS.

Table 1

Stability of pooled cryopreserved blood from five donors over a period of 4 months

\begin{tabular}{llll}
\hline $\begin{array}{l}\text { Day after } \\
\text { freezing }\end{array}$ & $\begin{array}{l}\text { Mean } \\
\text { absorbance } \\
\text { saline control }\end{array}$ & $\begin{array}{l}\text { Mean } \\
\text { absorbance } \\
0.5 \mathrm{EU} / \mathrm{ml}\end{array}$ & $\begin{array}{l}\text { Reactivity } \\
\text { (\% saline } \\
\text { control) }\end{array}$ \\
\hline 0 & $0.045 \pm 0$ & $0.311 \pm 0.06$ & 691 \\
40 & $0.068 \pm 0.01$ & $0.298 \pm 0.02$ & 439 \\
118 & $0.077 \pm 0.01$ & $0.755 \pm 0.05$ & 980 \\
\hline
\end{tabular}

Blood was stimulated with $0.5 \mathrm{EU} / \mathrm{ml}$ LPS from $E$. coli 0113 overnight, $n=4$. $\mathbb{L}-1 \beta$ measured in the supernatants by ELISA is shown as absorbance.

\subsection{Comparison of the reactivity of cryopreserved with fresh whole blood}

The reactivity of the cryopreserved blood to endotoxin stimulation was compared to that of fresh blood of the same individual donors. As can be seen in Fig. 7, $0.5 \mathrm{EU} / \mathrm{ml}$ LPS induced significant IL-1 $\beta$ release both in the fresh and the cryopreserved blood of every donor. This is the sensitivity limit of the most sensitive rabbit strain for testing according to the European Pharmacopoeia for injectable drugs.

We also compared the sensitivity of pooled fresh and cryopreserved blood from five donors to a doseresponse curve of LPS 0113 starting at $0.125 \mathrm{EU} / \mathrm{ml}$ (3 replicates). Both fresh and cryopreserved blood released statistically significant amounts of IL-1 starting at $0.5 \mathrm{EU} / \mathrm{ml}$ in the test sample $(p<0.01$ in both cases). The same was true for the release of IL- 6 and IL-8.

When the response to endotoxin stimulation of cryopreserved and fresh blood from the same donors was compared in a kinetic study, a noticeable differ- 
Table 2

Intra-aliquot variability of cryopreserved blood from one donor

\begin{tabular}{lclcccc}
\hline & $\begin{array}{l}\text { Aliquot } 1, \\
\text { saline }\end{array}$ & $\begin{array}{l}\text { Aliquot 2, } \\
\text { saline }\end{array}$ & $\begin{array}{l}\text { Aliquot 3, } \\
\text { saline }\end{array}$ & $\begin{array}{l}\text { Aliquot 4, } \\
0.5 \mathrm{EU} / \mathrm{ml}\end{array}$ & $\begin{array}{l}\text { Aliquot 5, } \\
0.5 \mathrm{EU} / \mathrm{ml}\end{array}$ & $\begin{array}{l}\text { Aliquot 6, } \\
0.5 \mathrm{EU} / \mathrm{ml}\end{array}$ \\
\hline Minimum & 0.040 & 0.043 & 0.048 & 0.242 & 0.216 & 0.384 \\
Median & 0.046 & 0.047 & 0.053 & 0.259 & 0.317 & 0.495 \\
Maximum & 0.107 & 0.051 & 0.066 & 0.339 & 0.490 & 0.582 \\
Mean & 0.053 & 0.047 & 0.054 & 0.274 & 0.327 & 0.085 \\
S.D. & 0.022 & 0.003 & 0.006 & 0.036 & 0.012 & 0.061 \\
S.E.M. & 0.008 & 0.001 & 0.002 & 0.012 & 26.08 & 12.26 \\
cv\% & 42.17 & 5.4 & 10.66 & 12.83 & 021 \\
\hline
\end{tabular}

Eight replicates from each aliquot of blood of the same donor were stimulated with LPS from $E$. coli 0113 overnight. IL-1 $\beta$ measured in the supernatants by ELISA is shown as absorbance.

ence in the kinetics of the LPS-inducible IL-1 $\beta$ release was observed (Fig. 8, upper panel). Measurable IL- $1 \beta$ release occurred with several hours delay in cryopreserved compared to fresh blood. This could be attributed to the presence of the cryoprotectant, since fresh blood containing $10 \%$ DMSO showed the same delay. Furthermore, in both cases, the presence of DMSO increased the maximum amount of IL- $1 \beta$ released sevenfold (fresh blood plus DMSO) and fivefold (cryopreserved blood). The same held true for IL-6 (Fig. 8, center panel), although here, the amount of IL-6 was increased nearly 20 -fold. TNF $\alpha$ release was no longer detectable after the addition of DMSO
(Fig. 8, lower panel), both in fresh and cryopreserved blood.

Next, the reactivity of cryopreserved blood to a variety of immune stimuli was tested in comparison to fresh blood. Different pyrogenic (fever-inducing) stimuli, including LPS, LTA and phytohaemagglutinin-L (PHA-L), induced IL-1 $\beta$ release in cryopreserved blood, but not the nonpyrogenic substances PHA-E, glucans and monophosphoryl lipid A (data not shown). Differences were seen for curdlan and zymosan A and, in terms of a higher sensitivity of the cryopreserved blood, for endotoxin from Pseudomonas aeruginosa. Taken together, the cryopreservation

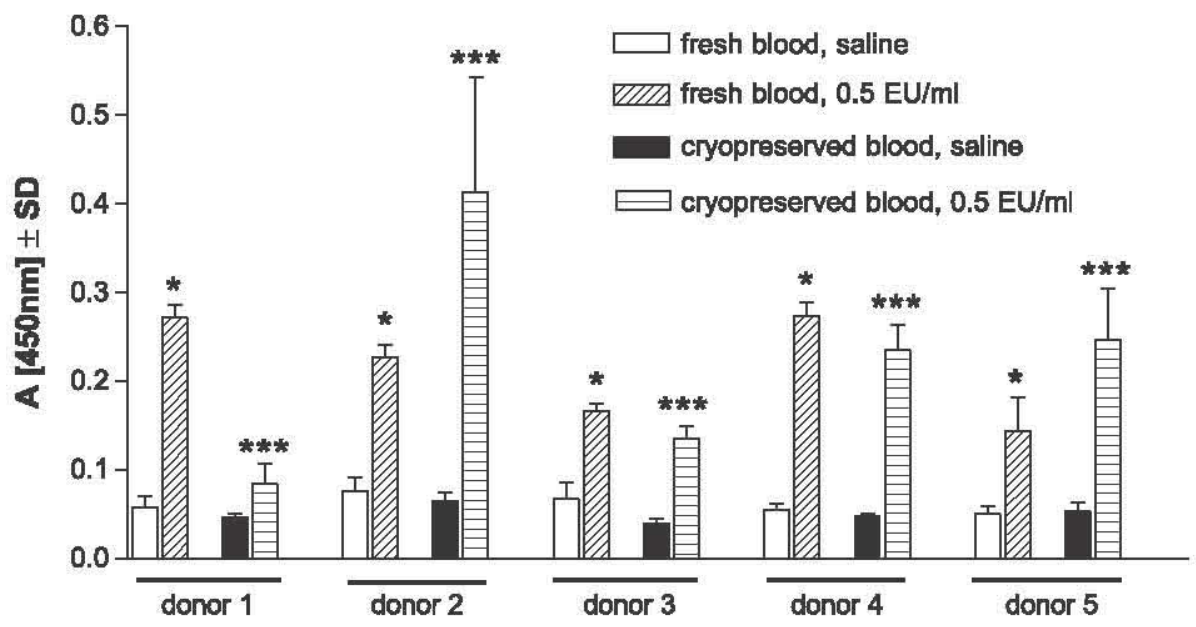

Fig. 7. Comparison of the reactivity of fresh and frozen blood of five separate donors. Fresh blood (four replicates each) and thawed cryopreserved blood (six replicates each, saline control $n=8$ ) from the same five donors were stimulated with LPS from $E$. coli O113 overnight. $\mathbb{L}-1 \beta$ measured by ELISA is given as absorbance value \pm S.D. Cryopreserved blood was thawed immediately after complete freezing and was incubated in parallel with the fresh blood of the same donors from the same blood donation (representative experiment of three). ${ }^{*} p<0.05$, ${ }^{* * *} p<0.001$, against the respective saline control ( $t$-test and Mann-Whitney posttest). 

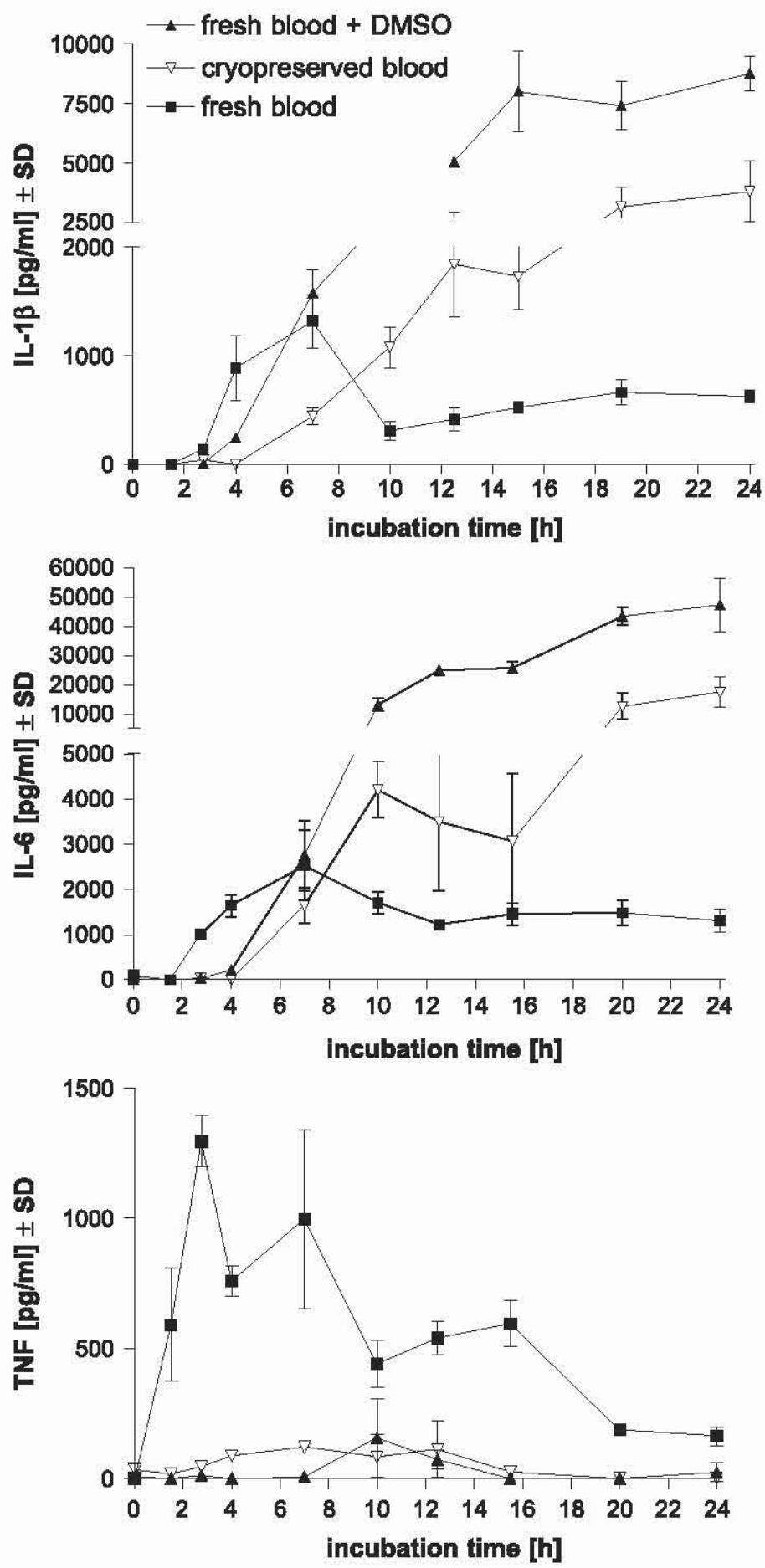

Fig. 8. Kinetics of cytokine response of fresh blood, fresh blood after addition of $10 \%$ DMSO and cryopreserved blood. Three replicates of blood samples pooled from five donors were challenged with $1.0 \mathrm{EU} / \mathrm{ml}$ LPS from E. coli $\mathrm{O} 111$ for the times indicated. Cytokines were measured by ELISA and are given as $\mathrm{pg} / \mathrm{ml} \pm$ S.D. 
Table 3

Interference testing of clinical-grade parenterals in fresh and cryopreserved blood

\begin{tabular}{lllcll}
\hline Trade name & Drug & ELC (EU/ml) & MVD & MID cryoblood & MID fresh blood \\
\hline Lasix & furosemide-sodium & 15 & 30 & $1 / 10$ & $1 / 30$ \\
Ultracain & articain/epinephrine & 75 & 150 & $1 / 150$ & Not testable \\
Binotal & ampicillin-sodium & 75 & 150 & $1 / 50$ & $1 / 100$ \\
Bronchoparat & theophyline & 37.5 & 75 & $1 / 25$ & $1 / 75$ \\
Fenistil & dimethindenmaleate & 93.75 & 187.5 & $1 / 150$ & $1 / 180$ \\
Sostril & ranitidine & 75 & 150 & $1 / 20$ & $1 / 100$ \\
Beloc & metoprolotartrate & 75 & 150 & $1 / 40$ & $1 / 50$ \\
\hline
\end{tabular}

Drug samples were added to fresh blood of two donors $(n=4)$ or cryopreserved blood of one donor $(n=4)$ and spiked with $0.5 \mathrm{EU} / \mathrm{ml}$ LPS from E. coli 0113 overnight. $\mathbb{L}-1 \beta$ measured in the supernatants by ELISA is shown as absorbance. For positive spike retrieval, $50-200 \%$ of the response to the LPS spike in saline had to be found in the spiked drug sample. ELC, endotoxin limit concentration according to European Pharmacopoeia; MVD, maximum valid dilution (ELC/0.5 EU/ml); MD, minimum interference dilution.

procedure did not alter the spectrum of pyrogens or immune stimuli detected and did not cause the cryopreserved blood to react to substances which fresh blood does not react to either.

To test whether cryopreservation might interfere with the detection of contaminations in pharmaceuticals, e.g., as a result of hemolysis, the freezing/ thawing procedure or the DMSO, a series of drugs was tested with fresh and cryopreserved blood as to their interference with a given LPS spike. Results are summarized in Table 3.

The interference differed, though surprisingly, the cryopreserved blood proved to be less prone to interference than the fresh blood. The minimal interference dilutions were lower for the cryopreserved blood and always at or below the maximal valid dilution (MVD). These data show that a broad variety of drugs can be controlled by a pyrogen test based on cryopreserved blood, maintaining the endotoxin limit concentration (ELC) according to the pharmacopoeias established for the Limulus amoebocyte lysate assay without that test's restriction to Gram-negative LPS. However, interference testing must be performed in every case with both fresh and cryopreserved blood for any given drug.

\section{Discussion}

The utility of human whole blood assays has been demonstrated in a broad variety of applications. All applications so far have to be carried out within a few hours of blood withdrawal, which makes parallel processing difficult and leads to a high variability in clinical samples. This complicates donor pretesting and poses problems with regard to the continuous availability of fresh blood samples.

The procedure described here offers a continuous supply with a highly homogenous batch of blood. A regular blood donation $(500 \mathrm{ml})$ would suffice for 5000 tests (tube format) or 25,000 tests (microtiter plate format), which can be increased even further by pooling blood from several donors before freezing. Additionally, blood batches can be pretested with regard to sensitivity, and infections, such as HIV or hepatitis, can be excluded. The latter, however, can also be achieved by prescreening donors following the standard guidelines for blood donation.

The established procedure has been optimized with regard to the retention of sensitive cytokine response to pyrogenic contamination. Initial freezing attempts employing other cryoprotectants (hydroxyethylstarch, glycerol or propanediol) and other freezing procedures (conventional freezer, vapor phase of liquid nitrogen) have been unsuccessful to date. Both the freezing and the thawing protocol were optimized and the reactivity of the cryopreserved blood regarding IL$1 \beta$ release was compared to that of fresh blood. The inter- and intra-aliquot variability was tested. Despite variances in some experiments, the IL- $1 \beta$ response to $0.5 \mathrm{EU} / \mathrm{ml}$ was significantly different from the controls in each case. The reaction of cryopreserved blood to different pyrogenic and nonpyrogenic substances in comparison to the reaction of fresh blood was evaluated.

The differential blood cell counts of fresh versus cryopreserved blood showed that monocytes and lymphocytes were still viable after the cryopreserva- 
tion procedure, thus implying that functional assays of these cells can still be performed efficiently. In addition, the ratio of monocytes to lymphocytes remained unchanged. However, the surface properties of neutrophilic granulocytes were affected by the cryopreservation procedure, suggesting that neutrophil function may be lost. The established pooling procedure allows the preparation of large batches of cryopreserved blood and also reduces the risk of possible abnormal individual reactions. The sensitivity of the blood appears to be stable over a time period of at least 4 months.

Apart from other application possibilities, the results presented indicate that cryopreserved blood can be used as an alternative to fresh blood in the In vitro Pyrogen Test to detect contaminations in batches of different drugs. These first data show that a broad variety of drugs could be controlled using the cryopreserved blood, maintaining the endotoxin limit concentration (ELC) established for the Limulus amebocyte lysate assay without the restriction to Gram-negative LPS.

However, the hemolysis, dead PMN as well as the DMSO might result in interference with some drugs, e.g., drugs that bind to hemoglobin, necessitating product-specific validation. It is also possible that the synergy of LPS and hemoglobin influences the results. Therefore, the suitability of cryopreserved blood for pyrogen testing of a given product will have to be demonstrated by separate interference testing.

This approach may also find applications in highthroughput screening. It is tempting to base screening assays on homogeneous preparations of human primary cells, requiring no prior culture or isolation procedures. Given the broad variety of immunomodulators in clinical use, several application opportunities can be imagined. Since the whole blood model also allows the determination of eicosanoid release (von Aulock et al., 2003), this might extend to modulators of eicosanoid formation, such as nonsteroidal anti-inflammatory drugs (NSAID). However, the feasibility and relevance of this approach will have to be established.

Taken together, a variety of immunological models and tests might benefit from the availability of functional, cryopreserved blood. Efforts to make this available on a large scale are ongoing. The advantages and respective adaptations for different uses will have to be established. The approach promises to overcome problems of availability and standardization of human primary blood leukocytes and to provide standardized blood as an immunological reagent for a broad spectrum of applications.

\section{Acknowledgements}

This work was supported by the Bundesministerium für Bildung und Forschung (BMBF 11425A) and the Stiftung zur Förderung der Erforschung von Ersatz- und Ergänzungsmethoden zur Einschränkung von Tierversuchen (set). The procedure has been granted a European patent (97 122 072.8).

\section{References}

Cavins, J.A., Djerassi, I., Roy, A.J., Klein, E., 1965. Preservation of viable human granulocytes at low temperature in dimethylsulfoxide. Cryobiology 2, 129.

De Boer, M., Reijneke, R., Van de Griend, R.J., Loos, J.A., Roos, D., 1981. Large-scale purification and cryopreservation of human monocytes. J. Immunol. Methods 43, 225.

Fennrich, S., Wendel, A., Hartung, T., 1999. New applications of the human whole blood pyrogen assay (PyroCheck). ALTEX 16,146

Fenton, M.J., Golenbock, D.T., 1998. LPS-binding proteins and receptors. J. Leukoc. Biol. 64, 25.

Frim, J., Mazur, P., 1983. Interactions of cooling rate, warming rate, glycerol concentration, and dilution procedure on the viability of frozen-thawed human granulocytes. Cryobiology 20, 657.

Goldman, J.M., Th'ng, K.H., Park, D.S., Spiers, A.S., Lowenthal, R.M., Ruutu, T., 1978. Collection, cryopreservation and subsequent viability of haemopoietic stem cells intended for treatment of chronic granulocytic leukaemia in blast-cell transformation. Br. J. Haematol. 40, 185.

Handin, R.I., Valeri, C.R., 1972. Improved viability of previously frozen platelets. Blood 40, 509 .

Hartung, T., 2002. Comparison and validation of novel pyrogen tests based on the human fever reaction. Altern. Lab. Anim. 30 (Suppl. 2), 49.

Hartung, T., Wendel, A., 1995. Detection of pyrogens using human whole blood. ALTEX 12, 70.

Hartung, T., Docke, W.D., Gantner, F., Krieger, G., Sauer, A., Stevens, P., Volk, H.D., Wendel, A., 1995. Effect of granulocyte colony-stimulating factor treatment on ex vivo blood cytokine response in human volunteers. Blood 85, 2482.

Hartung, T., Doecke, W.D., Bundschuh, D., Foote, M.A., Gantner, F., Hermann, C., Lenz, A., Milwee, S., Rich, B., Simon, B., Volk, H.D., von Aulock, S., Wendel, A., 1999. Effect of filgrastim treatment on inflammatory cytokines and lymphocyte functions. Clin. Pharmacol. Ther. 66, 415. 
Hermann, C., von Aulock, S., Graf, K., Hartung, T., 2003. A model of human whole blood lymphokine release for in vitro and ex vivo use. J. Immunol. Methods 275, 69.

Knorpp, C.T., Merchant, W.R., Gikas, P.W., Spencer, H.H., Thompson, N.W., 1967. Hydroxyethyl starch: extracellular cryophylactic agent for erythrocytes. Science 157, 1312.

Morath, S., Geyer, A., Spreitzer, I., Hermann, C., Hartung, T., 2002. Structural decomposition and heterogeneity of commercial lipoteichoic acid preparations. Infect. Immun., Feb. 70 (2), 938.
Schumann, R.R., 1992. Function of lipopolysaccharide (LPS)binding protein (LBP) and CD14, the receptor for LPS/LBP complexes: a short review. Res. Immunol. 143, 11.

von Aulock, S., Boneberg, E.M., Hartung, T., 2000. Intermittent GCSF (filgrastim) treatment cannot induce lymphocytosis in volunteers. Clin. Pharmacol. Ther. 68, 104.

von Aulock, S., Hermann, C., Hartung, T., 2003. Determination of the eicosanoid response to inflammatory stimuli in whole blood and its pharmacological modulation ex vivo. J. Immunol. Methods 277, 53. 